\title{
Survey of lines in M 31: [CII] as SFR tracer at $\sim 50$ pc scales
}

\author{
M. J. Kapala ${ }^{1}$, K. Sandstrom ${ }^{1,2}$ and B. Groves ${ }^{1}$ \\ ${ }^{1}$ Max Planck Institut für Astronomie, Königstuhl 17, D-69117 Heidelberg, Germany \\ email: kapala@mpia.de, ${ }^{2}$ Steward Observatory, Tucson, USA
}

The [CII] $158 \mu \mathrm{m}$ line is typically the brightest far-IR emission line from star-forming galaxies. As such, this line is a possible tracer of star-formation, but to do so we need the relative contributions of different ISM phases. Using high physical resolution observations of the [CII] $158 \mu \mathrm{m}$ line from Herschel PACS in five 3' $\times 3$ ' field in M 31 and optical IFU spectra from $\mathrm{PPaK}$ and ancillary IR data, we are able to spatially separate out the ISM phases (Kapala et al. subm.). We find that a large fraction of [CII] emission in M 31 arises from diffuse gas $(\sim 20-90 \%)$, with a sub-linear relation of [CII]-SFR at $\sim 50$ pc scales. However, on $\sim \mathrm{kpc}$ scales, the observed empirical [CII]-SFR relation is in agreement with other extragalactic studies. The observed flattening of the fitted slope to the [CII]-SFR at $\sim 50 \mathrm{pc}$ scales might be explained by possible contributions to ISM gas heating by older stellar populations (ie. [CII] tracing longer timescales) and/or leaked photons from HII regions. The issue of leaked photons from HII regions should go away when averaged over larger scales $(>500 \mathrm{pc})$.
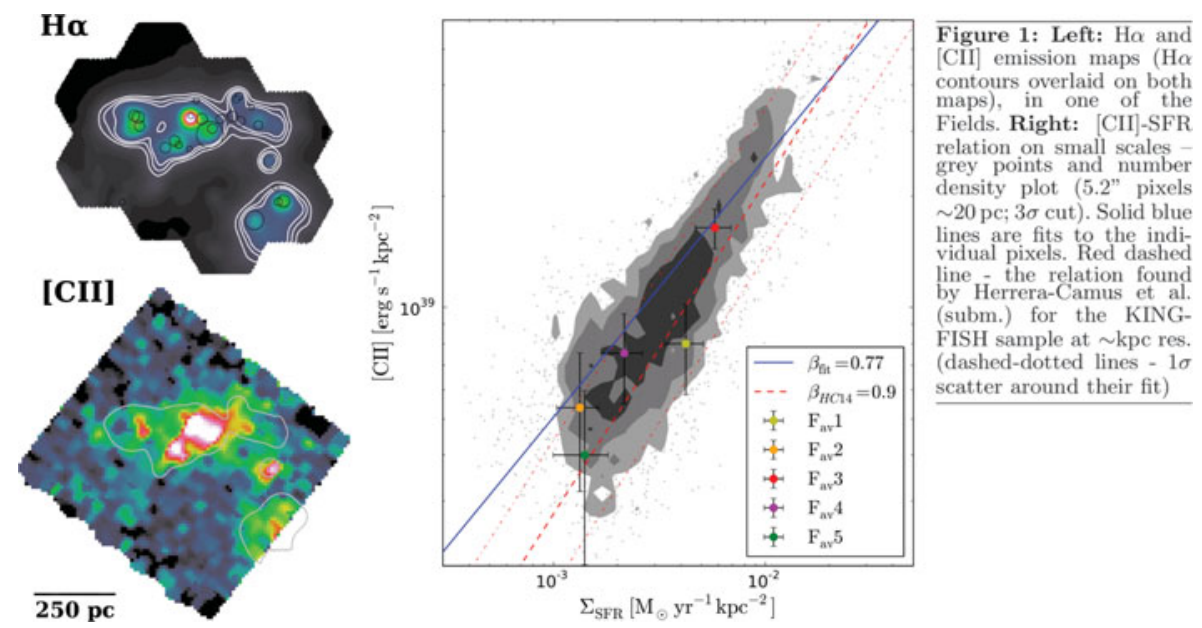

\section{Acknowledgements}

We acknowledge support from the DLR Grant 50OR1115.

Herschel is an ESA space observatory with science instruments provided by Europeanled Principal Investigator consortia and with important participation from NASA.

\section{Reference}

Kapala, M. J., et al., subm., ApJ 